\title{
Usefulness of position emission tomography/computed tomography in a case of sarcoidosis with multiorgan involvement
}

\author{
Norihiko Amano ${ }^{1}$, Soshi Takahashi ${ }^{1}$, Saori Hatachi ${ }^{1}$, and Shunichi Kumagai ${ }^{1}$ \\ ${ }^{1}$ Shinko Hospital
}

December 7, 2021

\begin{abstract}
Sarcoidosis, a systemic inflammatory disease of unknown etiology, can affect any site in the body. A bone lesion was unexpectedly detected by fluorodeoxyglucose position emission tomography/computed tomography (FDG PET/CT) in a patient with multiorgan sarcoidosis. FDG PET/CT should be considered for the detection of clinically silent lesions of sarcoidosis.
\end{abstract}

Title page

Usefulness of position emission tomography/computed tomography in a case of sarcoidosis with multiorgan involvement

Norihiko Amano, Soshi Takahashi, Saori Hatachi, and Shunichi Kumagai

The Center for Rheumatic Disease, Shinko Hospital, Kobe, Japan

Soshi Takahashi, M.D., Ph.D. (corresponding author)

Affiliation: The Center for Rheumatic Disease, Shinko Hospital, Kobe, Japan

Postal address: 1-4-47, Wakinohama-cho, Chuo-ku, Kobe, 651-0072, Japan

Telephone number: + 81782616711

Email address: takahashi.soshi@shinkohp.or.jp

Norihiko Amano, M.D.

Affiliation: The Center for Rheumatic Disease, Shinko Hospital, Kobe, Japan

Email address: amano.norihiko@shinkohp.or.jp

Saori Hatachi, M.D., Ph.D.

Affiliation: The Center for Rheumatic Disease, Shinko Hospital, Kobe, Japan

Email address: hatachi.saori@shinkohp.or.jp

Shunichi Kumagai, M.D., Ph.D.

Affiliation: The Center for Rheumatic Disease, Shinko Hospital, Kobe, Japan

Email address: kumagais@kobe-u.ac.jp

Data availability statement 
Data sharing is not applicable to this article as no datasets were generated or analyzed during the current study.

\title{
Funding statement
}

Not applicable.

\section{Conflict of interest disclosure}

None declared.

\section{Ethics approval statement}

The ethic approval was not necessary for this case report. The patient's data and images are de-identified.

\section{Patient consent statement}

Written informed consent was obtained from the patient for publication of this case report and accompanying images.

\section{Key Clinical Message}

Sarcoidosis can affect any site in the body, and the clinical course is extremely variable and often asymptomatic. FDG PET/CT is useful in the diagnosis and workup of sarcoidosis, especially for bone lesions.

\begin{abstract}
Sarcoidosis, a systemic inflammatory disease of unknown etiology, can affect any site in the body. A bone lesion was unexpectedly detected by fluorodeoxyglucose position emission tomography/computed tomography (FDG PET/CT) in a patient with multiorgan sarcoidosis. FDG PET/CT should be considered for the detection of clinically silent lesions of sarcoidosis.
\end{abstract}

\section{Case presentation}

A 48-year-old female noticed a subcutaneous nodule. She presented with remittent fever, fatigue, weight loss, and abdominal pain. Laboratory tests showed elevated levels of lysozyme, soluble interleukin-2 receptor, and angiotensin-converting enzyme. Computed tomography (CT) revealed pulmonary reticular opacities, hepatomegaly, and massive splenomegaly. Fluorodeoxyglucose position emission tomography (FDG PET)/CT showed FDG uptake not only in the liver, spleen, and systemic lymph nodes, but also in the humerus, scapula, the 4-7th thoracic vertebrae, pelvis, and femur (Figure 1,2). Histological samples from the skin, liver, and bone marrow biopsies exhibited noncaseating granulomas with epithelioid cells, which were diagnosed as multiorgan sarcoidosis.

Sarcoidosis is a systemic inflammatory disease of unknown etiology characterized by the formation of noncaseating granulomas in the affected organs. Although FDG PET/CT is not included in the standard workup for sarcoidosis, its usefulness in the diagnosis of this condition and its subsequent management has been proposed (1). Moreover, FDG PET/CT has been reported to be especially useful in detecting bone lesions because it is difficult to detect bone sarcoidosis involvement using conventional radiography (2). Sarcoidosis can affect any site in the body while being asymptomatic. Therefore, FDG PET/CT should be considered for the detection of affected lesions of sarcoidosis.

\section{ACKNOWLEDGMENT}

We are grateful to the healthcare professionals who managed this patient. The authors would like to thank MARUZEN-YOSHUDO Co., Ltd. (https://kw.maruzen.co.jp/kousei-honyaku/) for the English language editing.

\section{AUTHOR CONTRIBUTIONS}

ST and TA contributed to the writing of this case and to the acquisition of the respective images. SH and KS reviewed and edited the manuscript. 


\section{REFERENCES}

1. Akaike G, Itani M, Shah H, et al. PET/CT in the diagnosis and workup of sarcoidosis: focus on atypical manifestations. RadioGraphics. 38:1536-1549, 2018.

2. Demaria L, Borie R, Benali K, et al. 18F-FDG PET/CT in bone sarcoidosis: an observational study. Clin Rheumatol. 39:2727-2734, 2020.

(A)

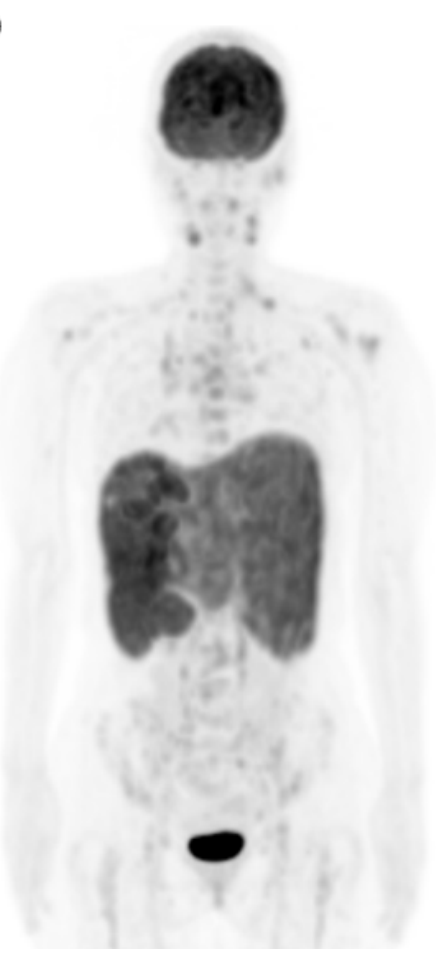

(B)

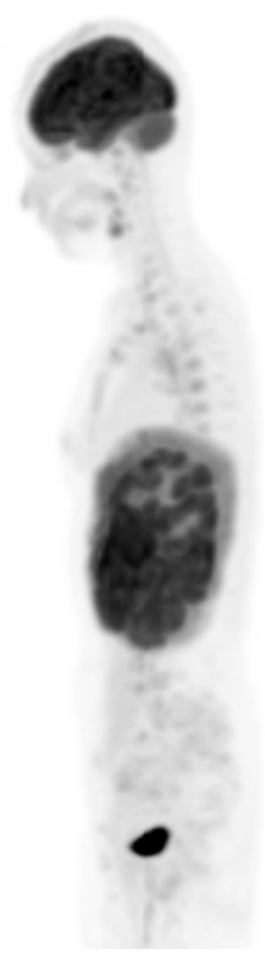

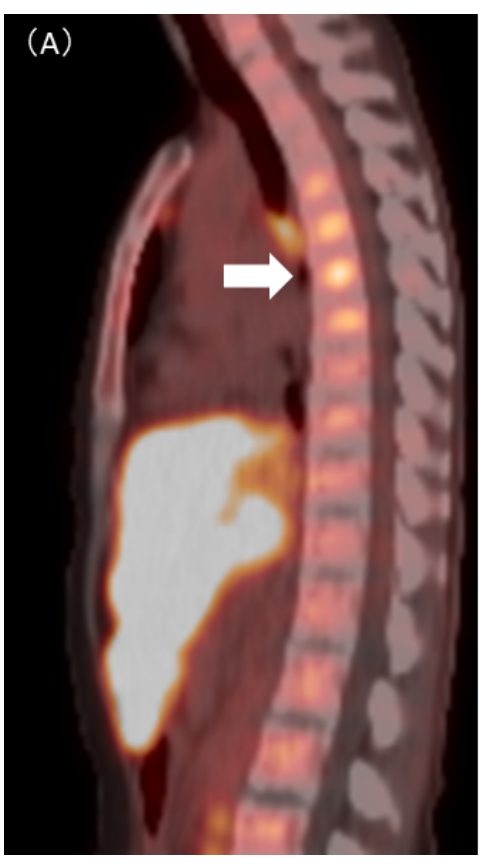
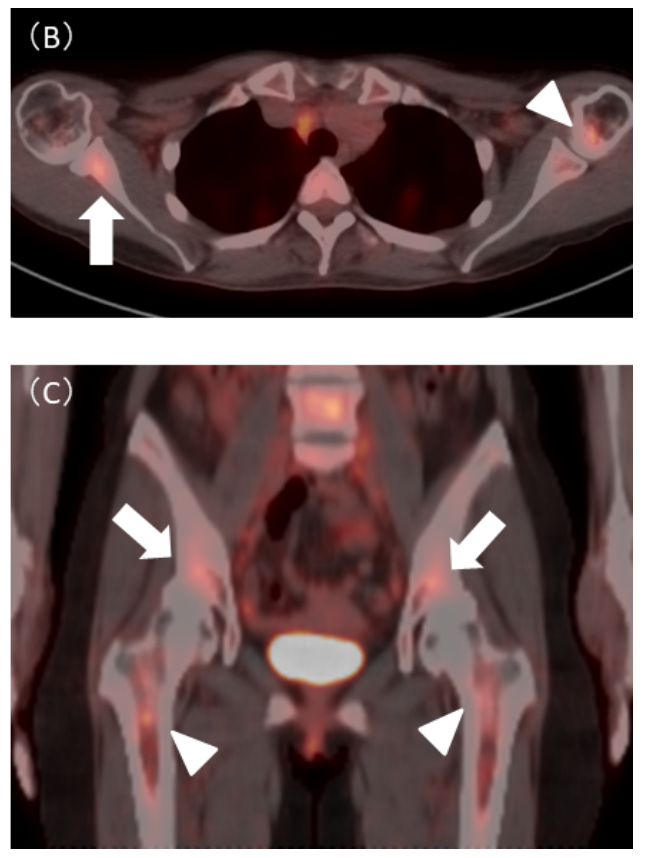